\title{
Mutants of Neurospora crassa Resistant to 8-Azaguanine
}

\author{
By G. R. HOFFMANN* AND H. V. MALLING \\ Mutagenesis Branch, National Institute of Environmental Health Sciences, \\ P.O. Box 12233, Research Triangle Park, North Carolina 27709, U.S.A.
}

(Received 8 January 1974; revised 7 March 1974)

\begin{abstract}
SUMMARY
Under conditions of limited-adenine supplementation, 8-azaguanine is a potent inhibitor of purine auxotrophic strains of Neurospora crassa. It is relatively ineffective in prototrophic strains. Spontaneous and u.v.-light-induced mutants, resistant to 8-azaguanine, were obtained in a strain carrying the ad-2, ad-3A, and ad-3B markers. The resistant mutants grew in the presence of $200 \mu \mathrm{g} \mathrm{8}$-azaguanine and $2.0 \mu \mathrm{g}$ adenine sulphate $/ \mathrm{ml}$ medium. Inhibition of the sensitive strain is virtually complete under these conditions. Higher adenine sulphate concentrations prevent inhibition of the sensitive strain by 8 -azaguanine. Resistance of the new mutants to 8-azaguanine was demonstrated by growth-tube experiments and by plating efficiencies in the presence and in the absence of the inhibitor. Growth-tube experiments were also used to study the reversal of 8-azaguanine induced inhibition by adenine sulphate. The resistance markers in the five strains studied were assigned to linkage group III. The locus designation $a z a-3$ was given to the resistance marker in a selected mutant of spontaneous origin. Resistance to 8-azaguanine is stable in the presence and in the absence of the inhibitor. In heterokaryons, 8-azaguanine resistance is recessive.
\end{abstract}

\section{INTRODUCTION}

The purine analogue 8-azaguanine (azaguanine) is a growth inhibitor in micro-organisms and in mammalian cells. It is incorporated into RNA, and possibly to a much lesser extent into DNA (Balis, I968; Harbers, Domagk \& Muller, 1968; Roy-Burman, 1970). The biological effects of azaguanine are generally attributed to interference with protein synthesis caused by modification of RNA (Harbers et al. 1968; Mahadevan \& Bhagwat, 1969). In addition to the formation of faulty RNA, the mechanism of action of azaguanine probably involves feedback inhibition of the production of normal guanylic acid (Balis, I 968). Conversion of the free base to azaguanylic acid is common to both mechanisms (Balis, I968). Effects of azaguanine on DNA and RNA synthesis have also been reported (Perevoschchikova, 1969; Rode \& Bayen, I 972).

Resistance to azaguanine has been reported in a variety of experimental organisms and is most frequently attributed to an alteration or loss of hypoxanthine-guanine phosphoribosyltransferase (HGPRT) activity (Balis, I968; Brockman, Sparks, Hutchinson \& Skipper, 1959; Harbers et al. 1968; Roy-Burman, 1970). Other reported mechanisms of resistance to azaguanine include specific loss of guanine phosphoribosyltransferase activity (Kalle \& Gots, 1961), decreased cell permeability to the analogue (Harbers et al. I968; Szybalski, Szybalska \& Ragni, I962), efficient deamination of azaguanine to the relatively inactive 8-azaxanthine (Harbers et al. 1968; Szybalski et al. 1962), and altered feedback inhibition of the purine biosynthetic pathway (Heslot, Nagy \& Whitehead, 1966; Shiio \& Ishii, 197I).

* Present address: Department of Biology, Meredith College, Raleigh, North Carolina 276ı, U.S.A. 
Selection for azaguanine resistance in Neurospora was reported by Bedair \& Fuerst (I96I), but resistant mutants were not genetically characterized. Jha (197I) later described the induction of 8-azaadenine resistant mutants at two loci in Neurospora. Several of the mutants were reported to be cross-resistant to azaguanine.

The present investigation describes mutants of Neurospora crassa which are resistant to the effects of azaguanine. These mutants are being used in the development of a new system for the detection of forward mutations (Hoffmann \& Malling, 1974).

\section{METHODS}

Strains. Mutants resistant to azaguanine were induced in a strain of Neurospora crassa (74-OR60-29A) with the following genetic markers: A, hist-2, ad-3A, ad-3B, nic-2, ad-2, inos. This strain, a strain (12-007-0073) with the A, ad-3B, al-2, cot, and pan-2 markers, and a strain carrying an mtr marker, were obtained from the culture collection of F. J. de Serres, National Institute of Environmental Health Sciences. Linkage studies were carried out with the alcoy multiple translocation stocks and follow-up testers developed by Perkins, Newmeyer, Taylor \& Bennett (1969). These strains were obtained from the Fungal Genetics Stock Center, Humboldt State College, Arcata, California, U.S.A.

Media. The plating medium used for colonial growth was Westergaard's basal medium (Westergaard \& Mitchell, 1947; de Serres \& Malling, 197I) containing 0.75\% L-sorbose, $0.05 \% \mathrm{D}$-glucose, and $0.05 \% \mathrm{D}$-fructose. For uninhibited mycelial growth and conidiation, Westergaard's or Fries basal medium (Horowitz \& Beadle, 1943; de Serres \& Malling, 197I) containing $\mathrm{I} \% \mathrm{D}$-glucose was used. When required, the following nutritive supplements were added to the medium: adenine sulphate ( $100 \mathrm{mg} / \mathrm{l}$ for full supplementation, $2 \mathrm{mg} / \mathrm{l}$ for limited adenine conditions), DL-histidine- $\mathrm{HCl}-\mathrm{H}_{2} \mathrm{O}(100 \mathrm{mg} / \mathrm{l})$, inositol $(30 \mathrm{mg} / \mathrm{l})$, nicotinamide (IO $\mathrm{mg} / \mathrm{l}$ ), D-calcium pantothenate (Io $\mathrm{mg} / \mathrm{l}$ ), L-tryptophan (I00 $\mathrm{mg} / \mathrm{l}$ ). The Difco Bacto-agar $(2.0 \%)$ used in solid media was washed three times in distilled water. Azaguanine was purchased from Schwarz-Mann Chemical Co. Stock solutions of $10 \mathrm{mg}$ azaguanine $/ \mathrm{ml}$ $\left(6.58 \times 1 \mathrm{IO}^{-2} \mathrm{M}\right)$ were prepared by dissolving the azaguanine in a minimal volume of $\mathrm{I} \mathrm{N}-\mathrm{NaOH}$ and diluting to the final volume with distilled water. Various amounts of filter-sterilized azaguanine were added to the medium immediately before use.

Neurospora techniques. The procedures followed in handling the experimental organism have been described in detail by de Serres \& Malling (1971).

Growth comparisons among strains and at different chemical concentrations were made in $20 \times 150 \mathrm{~mm}$ growth tubes, as previously described (Hoffmann, Malling \& Mitchell, 1973). Growth tubes were inoculated with single colonies isolated from plates. The point of furthest advance of the mycelial front was marked at $\mathrm{I} 2 \mathrm{~h}$ intervals while the tubes were incubated horizontally at $30{ }^{\circ} \mathrm{C}$. Measurements were made after the mycelial front reached the end of the tube. For plating-efficiency determinations, 200 conidia, determined by haemocytometer counts, were plated in Westergaard's plating medium under limited-adenine conditions. Colony formation in non-inhibitory medium was compared to that in medium containing $200 \mu \mathrm{g}$ azaguanine $/ \mathrm{ml}$. Colony counts after 3 days of incubation at $30^{\circ} \mathrm{C}$ were expressed as a percentage of the conidia plated.

All crosses were made in $20 \times 150 \mathrm{~mm}$ test tubes on supports made from Whatman No. I filter paper. Ten millilitres of Westergaard's basal medium containing $0.2 \%$ sucrose were dispensed per tube. The medium was supplemented for the nutritional requirements of the strains crossed. The crosses were incubated for 6 weeks at $25^{\circ} \mathrm{C}$. Preparation of ascospore suspensions was carried out by the procedure of F. J. de Serres (Hoffmann et al. 1973). 
Before plating, the ascospores were heat shocked in $0.15 \%$ purified agar for 50 min at $60{ }^{\circ} \mathrm{C}$. Ascospores were plated in Westergaard's plating medium, supplemented for the two parent strains. After two to three days' incubation at $35^{\circ} \mathrm{C}$, colonies were transferred to slants of Fries conidiating medium. All further tests were made with dilute suspensions of conidia grown on this medium. Auxotrophic markers were scored after 3 days' incubation at $25^{\circ} \mathrm{C}$ in Westergaard's liquid medium lacking only the nutrient being tested. Sensitivity or resistance to azaguanine was evaluated by a spot plate technique. A drop of conidial suspension was transferred on an applicator stick to one of eight spots on a Petri plate of supplemented plating medium containing $200 \mu \mathrm{g}$ azaguanine and $2 \mu \mathrm{g}$ adenine sulphate $/ \mathrm{ml}$. After 3 days' incubation at $30{ }^{\circ} \mathrm{C}$, resistance and sensitivity were scored by comparative growth on the spots.

Selection of mutants. Mutants were induced with u.v. light. Ultraviolet irradiation was performed with a $5 \mathrm{~W}$, No. Gi 5-T8 G.E. germicidal lamp. A dose of $2000 \mathrm{erg} / \mathrm{mm}^{2}$ was administered at the dose rate of $18.5 \mathrm{erg} / \mathrm{mm}^{2} / \mathrm{s}$, as measured with a Jagger meter (Jagger, I $96 \mathrm{I}$ ). Conidial suspensions were counted in a haemocytometer and adjusted to the concentration of $2 \times 10^{6}$ conidia $/ \mathrm{ml}$. Samples $(25 \mathrm{ml})$ were irradiated in $15 \times 100 \mathrm{~mm}$ glass Petri plates. The conidial suspensions were stirred on a magnetic stirrer during irradiation. Irradiation and plating were carried out in a room illuminated with red light in order to prevent photoreactivation. Mutants were selected in Westergaard's plating medium containing $200 \mathrm{mg}$ azaguanine and $2 \mathrm{mg}$ adenine sulphate $/ \mathrm{ml}$. Plates were incubated at $30{ }^{\circ} \mathrm{C}$ in the dark. Mutants that arose in the selection medium were made genetically homogenous by repeated single colony isolations from media containing azaguanine and limited adenine as above.

\section{RESULTS}

\section{Inhibition and mutant selection}

At $200 \mu \mathrm{g} / \mathrm{ml}$, azaguanine was effective in inhibiting colony formation when 200 prototrophic conidia were plated in $20 \mathrm{ml}$ medium. When $10^{6}$ conidia were plated in $20 \mathrm{ml}$ medium the background growth was heavy, particularly after 4 or 5 days of incubation. Inhibition of growth remained incomplete at azaguanine concentrations up to $500 \mu \mathrm{g} / \mathrm{ml}$. Higher concentrations were not tested.

When adenine auxotrophic conidia were plated in fully supplemented Westergaard's plating medium containing azaguanine, no inhibition was observed. Azaguanine becomes an effective inhibitor, however, at low adenine sulphate concentrations. Adenine sulphate concentrations as low as $0.5 \mu \mathrm{g} / \mathrm{ml}$ supported colony formation in Westergaard's plating medium. Inhibition of growth by $200 \mu \mathrm{g}$ azaguanine $/ \mathrm{ml}$ was virtually complete at adenine sulphate concentrations from 0.5 to $2 \cdot 0 \mu \mathrm{g} / \mathrm{ml}$. At higher adenine sulphate concentrations, inhibition by azaguanine was reversed.

Since azaguanine is a very effective inhibitor in an adenine auxotrophic strain under limited-adenine conditions, azaguanine resistant mutants were selected in Westergaard's plating medium containing $200 \mu \mathrm{g}$ azaguanine and $2.0 \mu \mathrm{g}$ adenine sulphate $/ \mathrm{ml}$. The level of background growth under these conditions is very low.

Azaguanine resistant mutants were obtained after u.v. irradiation at a dose of $2000 \mathrm{erg} / \mathrm{mm}^{2}$ ( $78 \%$ survival). Five strains which formed colonies of normal morphology in the presence and absence of azaguanine were selected for further study. These strains will be referred to as CI-8AG no. I, no. 2 , no. 4 , no. 6 , and no. Io. Strains CI-8AG no. I, no. 2 , no. 4 and no. Io were derived from mutants induced by u.v. light, while CI-8AG no. 6 arose as a spontaneous mutant. The colonies produced by the five azaguanine resistant strains in Westergaard's 

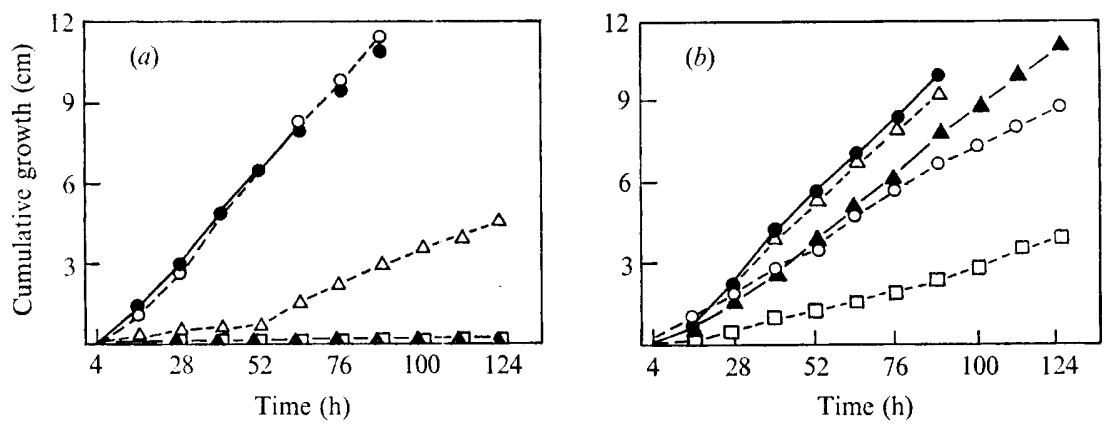

Fig. I. The effect of azaguanine on the sensitive strain and a resistant strain in growth tubes under limited-adenine conditions. (a) The sensitive strain, 74-OR60-29A; (b) CI-8AG no. 6. Azaguanine concentrations $(\mu \mathrm{g} / \mathrm{ml}): \bigcirc--\bigcirc, 0 ;-0,25 ; \triangle--\triangle, 50 ; \Delta-\Delta, 100 ; \square-\square, 200$.
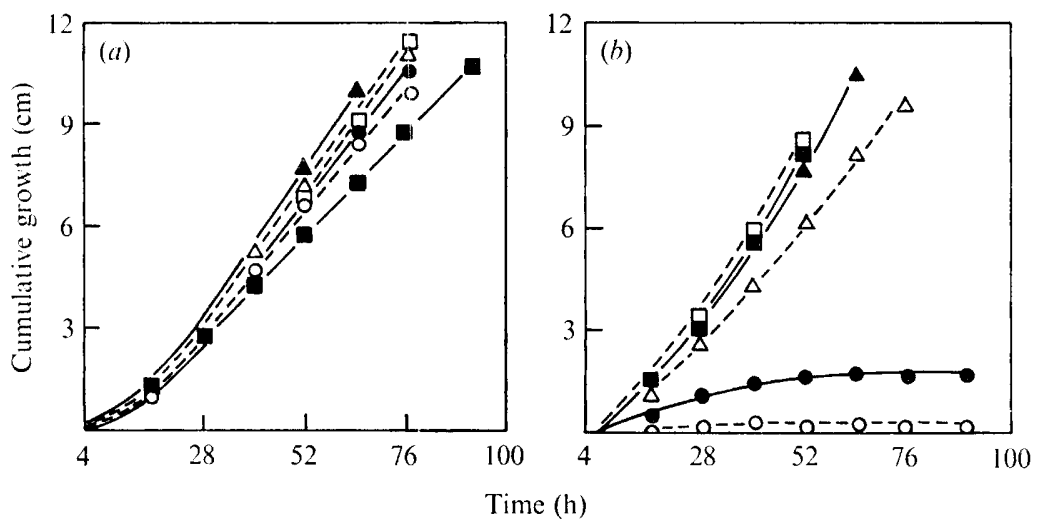

Fig. 2. Reversal of azaguanine-induced inhibition by adenine sulphate in the sensitive strain 74-OR60-29A. (a) Absence of azaguanine; $(b)$ presence of azaguanine $(200 \mu \mathrm{g} / \mathrm{ml})$. Adenine sulphate concentrations $(\mu \mathrm{g} / \mathrm{ml}): O--O, 2 ;--0,4 ; \Delta--\Delta, 8 ; \Delta-\Delta, \mathrm{I} 6 ; \square-\square, 32 ;$ - - 100.

plating medium without azaguanine were somewhat larger than those produced by the sensitive strain. The five CI-8AG strains grew and conidiated well on Fries conidiating medium.

\section{Growth studies}

Growth-tube experiments were used to demonstrate the resistance of the CI-8AG strains and to study the effect of adenine sulphate on inhibition by azaguanine. The advance of a mycelial front at several azaguanine concentrations was measured in Westergaard's conidiating medium at the adenine sulphate concentration of $2.0 \mu \mathrm{g} / \mathrm{ml}$. Results for the sensitive strain and for strain CI- $8 \mathrm{AG}$ no. 6 are presented in Fig. I, where each plotted point represents the mean of four growth tubes. Results for the four other resistant strains are the same as those for strain $\mathrm{CI}-8 \mathrm{AG}$ no. 6. Fig. 2 illustrates the effect of adenine sulphate on inhibition of the sensitive strain by azaguanine. The growth-tube data illustrate the resistance of the CI-8AG strains to azaguanine (Fig. I) and the reversal by adenine sulphate of the effects of azaguanine in the sensitive strain (Fig. 2).

In addition to the growth-tube experiments, resistance was evaluated by plating efficiency determinations. The data are presented in Table I. Each value represents the mean of three 
Table I. Plating efficiencies in the presence and absence of azaguanine under limited-adenine conditions

$\begin{array}{lcc}\text { Strain } & \text { No inhibitor } & \begin{array}{c}\text { Azaguanine } \\ (200 \mu \mathrm{g} / \mathrm{ml})\end{array} \\ \text { Sensitive (74-OR60-29A) } & 68 & 0 \\ \text { CI-8AG no. I } & 83 & 70 \\ \text { CI-8AG no. } 2 & 68 & 84 \\ \text { CI-8AG no. } 4 & 86 & 76 \\ \text { CI-8AG no. } 6 & 74 & 7 \mathrm{I} \\ \text { CI-8AG no. 10 } & 86 & 78\end{array}$

plates. The plating efficiencies indicate that the CI-8AG strains are resistant to azaguanine in plating medium under limited-adenine conditions.

The growth-tube and plating-efficiency experiments support one another in indicating that the new mutants are resistant to azaguanine under the specified conditions. The characteristics of the CI- $8 \mathrm{AG}$ strains remain unchanged after many transfers, and resistance is stable in cultures grown either in the presence or in the absence of azaguanine.

Cross-resistance evaluations were made by surface plating conidia on limited-adenine medium containing various concentrations of the inhibitor in question. Both the sensitive strain and the CI-8AG strains are sensitive to the effects of the pyrimidine analogues, 5fluorouracil and 5-fluorodeoxyuridine (FdUrd) at concentrations which do not inhibit FdUrd-resistant strains (Hoffmann et al. 1973). The amino acid analogue, DL-4-methyltryptophan, at a concentration of $110 \mu \mathrm{g} / \mathrm{ml}$, is inhibitory to the sensitive strain and to CI-8AG no. 6. Other CI-8AG strains were not tested. A methyltryptophan-resistant (mtr) mutant, which was used as a positive control, formed colonies in the presence of the inhibitor. At $25 \mu \mathrm{g} / \mathrm{ml}$, DL-4-methyltryptophan was partially inhibitory to both the sensitive strain and $\mathrm{CI}-8 \mathrm{AG}$ no. 6 . At concentrations as high as $200 \mu \mathrm{g} / \mathrm{ml}$, the adenine analogue 8-azaadenine was not sufficiently inhibitory to permit cross-resistance evaluations. Inhibition by the hypoxanthine analogue 6-mercaptopurine at a concentration of $200 \mu \mathrm{g} / \mathrm{ml}$ was incomplete. While CI-8AG no. 6 produced colonies of good morphology, growth of the sensitive strain was more restricted. Strain CI-8AG no. 6 is apparently cross-resistant to 6-mercaptopurine.

The ability of the CI-8AG strains to use hypoxanthine as their sole purine supply was checked in Westergaard's plating medium. Even at concentrations lower than $0.5 \mu \mathrm{g} / \mathrm{ml}$, colonies were formed.

\section{Genetic studies of the azaguanine resistant mutants}

Perkins' (Perkins et al. 1969) alcoy tester strains were used in the determination of the linkage groups of the resistance markers. Only adenine-auxotrophic progeny were scored for sensitivity or resistance because azaguanine resistance is not clearly expressed in prototrophic strains. Results from the alcoy cross indicated that the resistance marker in each of the five strains is linked to the ylo-I locus in the tester strain. This result indicated that the azaguanine-resistance gene is located in either linkage group III or linkage group VI because the tester strain includes a translocation involving these two linkage groups.

The standard follow-up tester strain recommended by Perkins (Perkins et al. 1969) was used to distinguish between linkage groups III and VI. This strain is marked by a tryptophan requirement (tryp-I) in linkage group III and the yellow locus (ylo-I) in linkage group VI. 
As in the previous series of crosses, only adenine auxotrophic progeny were tested for resistance. Results from this cross indicated linkage of the resistance marker in all five strains to tryp-I in linkage group III.

Jha (I97I) described 8-azaadenine resistant mutants, some of which are cross-resistant to the effects of azaguanine. Jha's mutants were mapped to two loci, designated $a z a-I$ and $a z a-2$, in linkage group I. The assignment of the resistance markers in the CI-8AG strains to linkage group III indicates that these genes are not the same as those reported by Jha.

The new azaguanine resistant mutant in strain CI-8AG no. 6 was given the locus designation $a z a-3$. Since the possibility of more than one azaguanine-resistance gene in linkage group III has not been tested, locus designations are not used for the other CI- 8 AG strains. Studies to map the $a z a-3$ gene within linkage group III, as well as to evaluate allelism in a series of azaguanine resistant mutants, are now in progress.

To determine whether the new genes are dominant or recessive, vegetative heterokaryons were formed. Conidia from a CI-8AG strain and a strain with the A, ad-3B, al-2, cot, and pan-2 markers were transferred to adenine-supplemented Fries conidiating medium lacking the supplementation for either homokaryotic strain but permitting the two strains to complement and grow as a heterokaryon. Conidia from heterokaryons of the CI-8AG strains did not form colonies in Westergaard's plating medium containing limited adenine $(2 \cdot 0 \mu \mathrm{g} / \mathrm{ml})$ and azaguanine $(200 \mu \mathrm{g} / \mathrm{ml})$. Although at high conidial concentrations background growth in the presence of azaguanine is slightly heavier in the CI-8AG-carrying heterokaryons than in fully sensitive heterokaryons, it was concluded that azaguanine resistance is recessive to sensitivity.

\section{DISCUSSION}

Reports from the literature vary considerably concerning the effectiveness of azaguanine as an inhibitor and the reversibility of its effects by adenine. While azaguanine concentrations of the order of I to $30 \mu \mathrm{g} / \mathrm{ml}$ are effective in mammalian tissue cultures (Chu, I97 $\mathrm{l}$; Chu, Brimer, Jacobson \& Merriam, 1969), higher concentrations are required for inhibition in fungi. A concentration of $200 \mu \mathrm{g} / \mathrm{ml}$ was found to be effective in Aspergillus (Morpurgo, I962) and in Schizosaccharomyces (Heslot et al. I966). Bedair \& Fuerst (I96I) reported that $350 \mu \mathrm{g}$ azaguanine $/ \mathrm{ml}$ completely inhibited growth of Neurospora. The relative ineffectiveness of azaguanine described here is closer to that found by Jha (197I).

The antagonism of azaguanine-induced inhibition by adenine sulphate is consistent with the findings of Morpurgo (I962) and Jha (I97I). Szybalski \& Smith (1959), on the other hand, found that adenine did not interfere with the effect of azaguanine in mammalian cells. Since adenine or hypoxanthine can serve as the sole purine source in adenine auxotrophic strains of Neurospora (Fairley \& Loring, 1949), it would be reasonable to expect that adenine reverses the inhibitory action of azaguanine by serving as a source of normal guanylic acid. The effect of adenine sulphate on inhibition could also be explained by competition for transport into the hyphae. That adenine can antagonize the uptake of guanine has been demonstrated in Schizosaccharomyces by Pourquie (1970).

In each $\mathrm{Cl}-8 \mathrm{AG}$ strain, the resistance marker was recessive to its sensitive allele. Incorporation of an aza gene into a heterokaryon, however, conferred upon the heterokaryon a slight decrease in sensitivity to azaguanine. Possible explanations are that the aza genes may be less than fully recessive, or that the inhibitory medium selects for a heterokaryon nuclear balance favouring the resistant component. Recessiveness of the azaguanine-resistance genes is consistent with reports for azaguanine resistance at other loci and in other organisms. The $a z a-I$ and $a z a-2$ genes in Neurospora are recessive in heterokaryons (Jha, I97I). Reces- 
siveness has also been demonstrated for azaguanine resistance in Chinese hamster cells in tissue culture (Chu et al. 1969) and in Aspergillus (Morpurgo, 1962).

The potential use of azaguanine sensitivity and resistance as a system for the study of chemical mutagenesis was suggested by Szybalski (1958). Morpurgo (1962) reported the use of azaguanine resistance for determining forward mutation frequencies in Aspergillus. Chu \& Malling (1968) developed a mutation system in which both forward mutation to azaguanine resistance and reversion to sensitivity can be detected in Chinese hamster cell cultures. The $a z a-3$ gene in strain CI- $8 \mathrm{AG}$ no. 6 is being used in the development of a new mutation detection system in a Neurospora heterokaryon (Hoffmann \& Malling, 1974).

This work was supported by a National Defense Education Act Title IV Fellowship, Oak Ridge Associated Universities, the Department of Botany of the University of Tennessee, and the National Institute of Environmental Health Sciences.

\section{REFERENCES}

Balis, M. E. (1968). Antagonists and nucleic acids. In Frontiers of Biology, vol. 10. Amsterdam: North Holland Publishing.

Bedair, B. J. \& Fuerst, R. (1961). Adaptability of Neurospora to 6-diazo-5-oxo-L-norleucine and related inhibiting agents. Antimicrobial Agents and Chemotherapy I, 260-267.

Brockman, R. W., Sparks, C, Hutchinson, D. \& Skipper, H. E. (1959). A mechanism of resistance to 8-azaguanine. I. Microbial studies on the metabolism of purines and 8-azapurines. Cancer Research 19, $177-188$.

CHU, E. H. Y. (197I ). Mammalian cell genetics. III. Characterization of X-ray-induced forward mutations in Chinese hamster cell cultures. Mutation Research II, 23-34.

Chu, E. H. Y., Brimer, P., Jacobson, K. B. \& Merriam, E. V. (1969). Mammalian cell genetics. I. Selection and characterization of mutations auxotrophic for L-glutamine or resistant to 8-azaguanine in Chinese hamster cells in vitro. Genetics 62, 359-377.

Chu, E. H. Y. \& Malling, H. V. (1 968). Mammalian cell genetics. II. Chemical induction of specific locus mutations. Proceedings of the National Academy of Sciences of the United States of America 6r, I3061312.

Fatrley, J. L. \& LoRING, H. S. (1949). Growth-promoting activities of guanine, guanosine, guanylic acid, and xanthine for a purine-deficient strain of Neurospora. Journal of Biological Chemistry 177, 451-459.

Harbers, E., Domagk, G. \& Muller, W. (1968). Introduction to Nucleic Acids - Chemistry, Biochemistry and Functions, pp. 294-328. New York: Reinhold Book Co.

Heslot, H., NAGY, M. \& WhITEHEAD, E. (1966). Recherches genetiques et biochimiques sur la primiere enzyme de la biosynthesis des purines chez le Schizosaccharomyces pombe. Comptes rendus hebdomadaire des séances de l'Académie des sciences 263, D57-58.

Hoffmann, G. R. \& Malling, H. V. (1974). Detection of 8-azaguanine resistant mutants in a Neurospora heterokaryon. Fifth Annual Environmental Mutagen Society Meeting Abstracts, Washington D.C., 8-1 I March 1974.

HoffmanN, G. R., Malling, H. V. \& Mitchell, T. J. (1973). Genetics of 5 -fluorodeoxyuridine resistant mutants of Neurospora crassa. Canadian Journal of Genetics and Cytology 15, 831-844.

Horowitz, N. H. \& Beadle, G. W. (1943). A microbiological method for the determination of choline by use of a mutant of Neurospora. Journal of Biological Chemistry 150, 325-333.

JAGGER, J. (1961). A small and inexpensive UV dose-rate meter useful in biological experiments. Radiation Research 14, 394-403.

JHA, K. K. (197I). Genes conferring resistance to 8-azaadenine in Neurospora crassa and the variability of resistant alleles in the aza-l locus with respect to purines. Molecular and General Genetics 114, $156-167$.

KalLE, G. P. \& Gots, J. S. (1961). Alterations in purine nucleotide pyrophosphorylases and resistance to purine analogues. Biochimica et biophysica acta 53, 166-173.

Mahadevan, P. R. \& Bhagwat, A. S. (1969). Inhibition of protein synthesis in Neurospora crassa by 8-azaguanine. Indian Journal of Biochemistry 6, 169-1 74. 
Morpurgo, G. (I962). A new method of estimating forward mutation in fungi: resistance to 8-azaguanine and p-fluorophenylalanine. Scientific Re'ports of the Instituto Superiore di Sanità 2, 9-1 2.

Perevoschchikova, K. A. (1969). Effect of 8-azaguanine on nucleic acid and protein synthesis in Ehrlich ascites Cancer cells in mice and in rat liver. Cancer Chemotherapy Abstracts no, 108.

Perkins, D. D., Newmeyer, D., Taylor, C. W. \& Bennett, D. C. (1969). New markers and map sequences in Neurospora crassa, with a description of mapping by duplication coverage, and of multiple translocation stocks for testing linkage. Genetica 40, 247-278.

Pourquie, J. (1970). Antagonism by adenine in the nutrition of Schizosaccharomyces pombe mutants. Inhibition at the level of guanine uptake. Biochimica et biophysica acta 209, 269-277.

Rode, A. \& BAYEN, M. (1972). Action de la 8-azaguanine sur la division cellulaire de Chlorella pyrenoidosa. I. Inhibition selective de la synthese de l'ADN nucleare. Planta 102, 237-246.

Roy-Burman, P. (1970). Analogues of nucleic acid components - mechanisms of action. In Recent Results in Cancer Research, vol. 25. New York: Springer-Verlag.

de Serres, F. J. \& Malling, H. V. (I97I). Measurement of recessive lethal damage over the entire genome and at two specific loci in the ad-3 region of a two-component heterokaryon of Neurospora crassa. In Chemical Mutagens: Principles and Methods for Their Detection, vol. 2, pp. 31 I-342. Edited by A. Hollaender. New York: Plenum Press.

SHIIO, I. \& IsHII, K. (197I). Genetically altered repression pattern of purine nucleotide synthesizing enzymes and inosine production in 8-azaguanine resistant mutants of Bacillus subtilis. Journal of Biochemistry 69, 339-347.

SzybaLSKi, W. (1958). Resistance to 8-azaguanine, a selective marker for a human cell line. Microbial Genetics Bulletin $\mathbf{1 6}, 30$.

Szybalski, W. \& SMith, M. J. (1959). Genetics of human cell lines. I. 8-Azaguanine resistance, a selective 'single-step' marker. Proceedings of the Society for Experimental Biology and Medicine ror, 662-666.

Szybalski, W., Szybalska, E. H. \& RaGNI, G. (1962). Genetic studies with human cell lines. National Cancer Institute Monographs 7, 75-89.

Westergaard, M. \& Mitchell, H. K. (I947). Neurospora. V. A synthetic medium favoring sexual reproduction. American Journal of Botany 34, 573-577. 